Research Article

\title{
Efficacy of transversus abdominis plane (TAP) block as part of multimodal analgesia after cesarean section delivery
}

\begin{abstract}
Background: Patients after Cesarean Section go through post operative pain. Most of this pain is experienced after the abdominal wall incision. Transversus abdominis plan (TAP) block as part of multimodal analgesia is a novel approach for patients delivered by cesarean section (CS). This technique (TAP block) lends itself particularly well to resource-poor settings as it does not require a nerve-stimulator. The aim of this study was to evaluate the efficacy of transversus abdominis plane (TAP) block when it is used as part of multimodal analgesia on obstetric patients after Cesarean Section.
\end{abstract}

Methods: Institution based prospective cohort study design was conducted on patients, who have Cesarean Section from February to May, 2015. All patients operated under spinal anesthesia for cesarean delivery were included. Patients divided into TAP block $(n=20)$ and controls $(n=20)$. The TAP block group was given bilateral $20 \mathrm{ml}$ of $0.25 \%$ bupivacaine at the end of surgery. Postoperative pain was assessed within the first 24 hours i.e. at 2 hours, 4 hours, 6 hours, 12 hours, and 24 hours using $100 \mathrm{~mm}$ long visual analogue scale (VAS), total analgesic consumption and time for the first analgesic request.

Result: There was reduction of VAS scores within the first 24 hours after cesarean section in TAP block group compared with the control group. VAS scores as median(IQR) at 2 hours $0.00(0.00-11.50)$ vs $39.00(7.75-60.50), \mathrm{p}=0.001$, at 4 hours $0.00(0.00-12.75)$ vs $17.50(13.25-24.00), \mathrm{p}=0.000$, at 6 hours $9.00(1.50-12.00)$ vs $22.00(14.75-45.00), \mathrm{p}=0.000$, at 12 hours $13.00(11.00-16.00)$ vs $42.00(10.00-52.00), \mathrm{p}=0.003$, at 24 hours $10.00(8.00$ $12.00)$ vs $15.50(11.25-26.00), \mathrm{p}=0.013$ respectively. The total analgesic Tramadol consumption within 24 hours was reduced in TAP block group, and time for the first analgesic request was significantly prolonged $(286.00 \mathrm{vs} 76.25, \mathrm{p}=0.000)$ minutes.

Conclusion: Bilateral TAP block provides lower postoperative severity of pain, reduced total postoperative Tramadol analgesics consumption and prolonged time for the first analgesic request after cesarean section under spinal anesthesia when it is used as multimodal analgesia. We recommend TAP block should be included as part of multimodal analgesia in the postoperative period for women after Cesarean Section delivery.

Keywords: Cesarean Section; Transversus abdominis plane block; postoperative analgesia; Spinal anaesthesia
Volume 3 Issue 3 - 2015

\section{Fentahun Tarekegn,' Setegn Eshetie, ${ }^{2}$ Kassaw Moges $^{3}$ \\ 'Department of Anesthesia, Debre Tabore University, Ethiopia ${ }^{2}$ Department of Microbiology, University of Gondar, Ethiopia ${ }^{3}$ Department of Anesthesia, Bahir Dar University, Ethiopia}

Correspondence: Fentahun Tarekegn, Department of Anesthesia, Debre Tabore University, Ethiopia, Tel +251913824072, Email tarekegnfentahun@gmail.com

Received: October 10,2014 | Published: November 12, 2015

\section{Introduction}

Cesarean section (CS) is the Universal surgical procedure which accounts $15 \%$ of birth world wide and $21.1 \%$ in the developed countries. ${ }^{1}$ Similarly, it is the common practice in our hospital, Ethiopia. The pain after surgery is experienced by means of patients below umbilicus surgery which derives from the abdominal wall incision. ${ }^{2}$ Transversus abdominis plan (TAP) block is the thriving regional analgesia practice for postoperative pain management with significant outcome on assisting infant care, early ambulation, and hindrance of postoperative morbidity. ${ }^{3}$ Opioids and non steroidal anti inflammatory drugs are regular postoperative pain relief in our setup. However, systemically administered opioids are causing adverse effects of sedation, nausea, vomiting, urinary retention, respiratory depression, delayed recovery, and prolonged postoperative ileus. ${ }^{4,5}$ On the other hand, TAP block analgesic technique can lower severity of pain, nausea and vomiting and paralytic ileus at post operative period. It is also having in reduction of postoperative morbidity, duration of hospitalization and hospital costs ${ }^{6}$ Even though, the epidural patientcontrolled analgesia (PCA) is given through a catheter placed into the epidural space, it has a chance of serious complications of epidural abscess, meningitis, vertebral canal hematoma, spinal cord ischemia and paraplegia. ${ }^{7,8}$

Studies showed on the TAP block which has been implemented to minimize systemic opioid drug complications as well as management of the postoperative pain effectively. ${ }^{9-12}$ TAP is a new, rapidly expanding regional anesthesia technique that provides analgesia to the parietal part of peritoneum as well as the skin and muscles of the anterior abdominal wall. ${ }^{13,14}$ It involves a single large bolus injection of local anesthetic into TAP anatomical compartment to block somatic afferent nerves on the anterior abdominal wall of T7 to L1 dermatomes. ${ }^{2,10,15}$

The blind (appreciating double pop) TAP block technique has comparable effect to an ultrasound guided procedure which has been performed under direct vision [16]. This type of block reduces the requirement of postoperative opioids use, increases the time for the first analgesic request, and provides helpful pain relief. ${ }^{17-19}$ In Ethiopia, most referral teaching hospitals and our Debre Tabor general hospital, the blind type of TAP block is practicing for the purpose of training postgraduate Msc anesthesia students and Bsc anaesthetists 
as a multimodal analgesia after cesarean section delivery. We did prospective study by considering the benefit of TAP that lends itself particularly well to resource-poor settings like in our most hospitals as it does not require a nerve-stimulator. Therefore, we assessed the efficacy of TAP block procedure with bupivacaine local anaesthetic after Cesarean Section delivery and finally to disseminate this practice to others of anesthesia professionals. In addition, it could be used as base line information for further research.

\section{Methods}

After obtaining Ethical approval from Amhara Regional Health Bureau Research Ethics Review Committee (RERC) with official permission letter to conduct the research and informed consent from each patient at preoperative period, a prospective cohort study design was conducted at Debre Tabor hospital North West Ethiopia from February to May 2015. All consecutive cesarean section patients at postoperative period were included by fulfilling the inclusion criteria of ASA status I - II patients undergoing Cesarean Section upon spinal anesthesia, bilateral $20 \mathrm{ml}$ of $0.25 \%$ bupivacaine was given at the end of Surgery. There was cases rejected as exclusion criteria of BMI $>35 \mathrm{~kg} / \mathrm{m}^{2}$, abuse of drugs or alcohol, allergies to local anaesthetics, refused mothers, sedation, infection at site of needle injection, and bleeding disorder.

The bilateral blind techniques of TAP block were performed with the patient in the supine position and palpating the iliac crest from anterior to posterior till latissimus dorsi muscle is appreciated. The triangle of Petit was easily appreciated at this position. Using a blunt needle, the skin was pierced just cephalic to the iliac crest along the midaxillary line over the triangle of Petit. The needle was advanced perpendicular to the skin, to appreciate a double pop sensation as the external oblique and internal oblique muscle layers were passed respectively. Subsequently, bilateral $20 \mathrm{~mL}$ of $0.25 \%$ bupivacaine were deposited following repeated aspiration to rule out vascular injection. All patients with TAP block were performed after wound dressing, and before extubation. Group of patients to cases and controls were divided randomly according to the plan of self-determining anaesthetists. Patients who got the block were considered as cases (TAP) and patients managed without the block were considered as controls (non TAP). TAP group patients were the bilateral block with a calculated safe dose of $20 \mathrm{ml}$ of $0.25 \%$ bupivacaine done after completion of the procedure. Non TAP group patients were managed only by systemic analgesics. One of the blinded data collectors recorded the intraoperative information. The other data collector who was also blinded for group allocation assessed the presence and severity of pain, total analgesic consumption and time for the first analgesic request. We made detaching of anesthetic note sheet to avoid the bias on identification of types of group till data collection was finished. The pain assessments were performed at 2, 4, 6,12 and 24 hours at rest, in the first $24 \mathrm{hr}$ postoperative period. Pain severity with patients at rest were measured using a VAS $(0=$ No Pain, $10=$ Worst Imaginable pain). The VAS were determined by the patient making a mark of their pain intensity on a line which is 100 millimetre long. The primary outcome variable is postoperative pain which was assessed using visual analogue scale VAS scores at 2, 4, 6, 12, 24 hours, total postoperative analgesics consumption within 24 hours and Time for the first analgesic request. The secondary outcome variables included as age, height, weight, body mass index (BMI), American Society of anaesthesiologists (ASA), and duration of Surgery. VAS is the Visual Analogue Scale which is a method of pain assessment determined by the patient making a mark of their pain intensity on a line which is $100 \mathrm{~mm}$ long.

\section{No pain—worst imaginable pain}

Time for the first analgesic request is the initial time in which patients need pain treatment. ${ }^{20} \mathrm{ASA}$ is the risk assessment of surgical patients according to the American society of Anesthesiologists group. The sample size (n) is determined on the basis of the mean 24 postoperative hours VAS Scores of the cases and controls as calculated from the previous study with mean $\pm \mathrm{SD}=(1.7 \pm 1.7$ vs $3.1 \pm 1.5) \mathrm{mg}$, $\mathrm{P}<0.05$ respectively. ${ }^{9}$ It was determined based on the comparison Mean formula which is $\mathrm{n}=(\mathrm{s} 12+\mathrm{s} 22) \mathrm{f}(\mathrm{a}, \mathrm{b}) /(\mathrm{m} 1-\mathrm{m} 2) 2 .{ }^{21}$ Where $\beta=$ $20 \%, \alpha=5 \%, \mathrm{~m} 1, \mathrm{~S} 1$ and M2, S2 are the mean and standard deviation of cases and controls respectively. Each number of case and control was 20 .

Data were analysed by using SPSS version 20. Demographic data were analyzed using Student's $t$ test (for normal distribution variables) and Fisher's exact test (for categorical variables). The data were tested for normality using the Shapiro-Wilk normality test. There was homogeneity of variance assessed by Levene's Test for equality of Variances. Therefore, an independent t-test was run on the normally distributed data for the time of first analgesic request. Since the repetitive VAS measurement and total analgesic consumption were not normally distributed, we run non parametric Mann-Whitney $\mathrm{U}$ test. The comparisons of categorical parameters were analyzed by using Fisher's exact test. Normal distributed data were presented as mean $\pm \mathrm{SD}$, not normally distributed data were presented as median (IQR), and categorical data were presented frequencies (percentages). A $\mathrm{p}$ value $<0.05$ considered as statistically significant.

\section{Results}

\section{Socio- demographic characteristics of the patients}

Forty patients operated for Cesarean Section upon under spinal anesthesia were included in this study. Of these, 20 were given bilateral TAP block with $20 \mathrm{ml}$ of $0.25 \%$ bupivacaine and 20 were without TAP block but managed by standard postoperative systemic analgesics. Demographic characteristics are comparable (Table 1). There was not any patient excluded from the study with criteria.

Table I Demographic characteristics of patients who underwent Cesarean Section in the period of February to May 2015.

\begin{tabular}{|c|c|c|}
\hline Cases (TAP) & Controls & $P$ value \\
\hline Age & $26.10 \pm 3.99 *$ & $26.40 \pm 3.88^{*}$ \\
\hline Height & $164.75 \pm 4.0 *$ & $160.70 \pm 3.52 *$ \\
\hline Weight & $58.50 \pm 6.21 *$ & $53.55 \pm 5.45 *$ \\
\hline BMI & $21.96 \pm 2.26 *$ & $20.6 I \pm 2.21 *$ \\
\hline Duration of Surgery & $43.50 \pm 7.45^{*}$ & $45.00 \pm 7.60 *$ \\
\hline ASA status & $16 / 20=80 \%$ & $17 / 20=85 \%$ \\
\hline II & $4 / 20=20 \%$ & $3 / 20=15 \%$ \\
\hline
\end{tabular}

Category reference $*$ mean $\pm \mathrm{SD}$

\section{Postoperative pain on VAS scores}

There was a different VAS scores at each time intervals of 24 postoperative hours at rest.VAS scores in TAP block and control group as median (IQR) at 2 hours $0.00(0.00-11.50)$ vs $39.00(7.75$ $60.50), \mathrm{p}=0.001$, at 4 hours $0.00(0.00-12.75)$ vs $17.50(13.25-24.00)$, $\mathrm{p}=0.000$, at 6 hours $9.00(1.50-12.00)$ vs $22.00(14.75-45.00), \mathrm{p}=0.000$, at 12 hours $13.00(11.00-16.00)$ vs $42.00(10.00-52.00), \mathrm{p}=0.003$, at 24 hours $10.00(8.00-12.00)$ vs $15.50(11.25-26.00), \mathrm{p}=0.013$ respectively. 


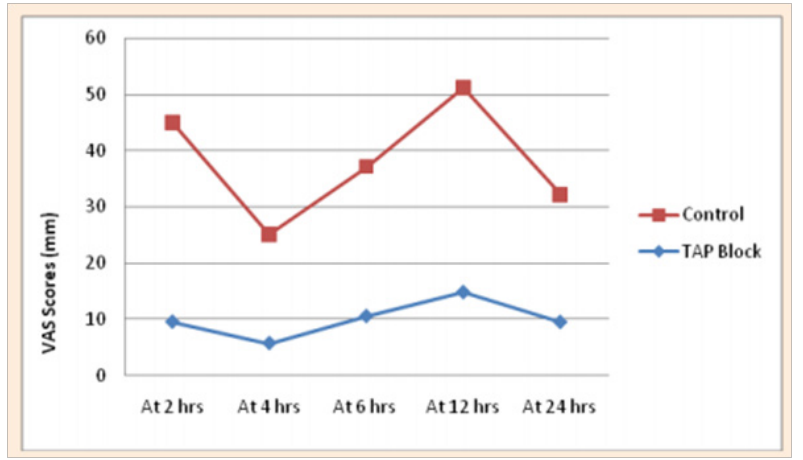

Figure I Mean post operative Visual Analogue scale (VAS) pain scores at rest in each group over the first 24 hours at Debre Tabore Hospital in the period of February to May, 20I5, North West Ethiopia.

\section{Postoperative analgesics consumption}

Tramadol and diclofenac consumption was checked by Shapiro Wilk test within 24 postoperative hours. Then Mann Whitney U test was used for association. Patients with TAP block showed reduced total Tramadol consumption as compared with controls i.e. TAP vs control was $975 \mathrm{mg}$ and $2025 \mathrm{mg}$ respectively within the first 24 postoperative hours, $p=0.001$. However, total diclofenac consumption (TAP vs control) $\mathrm{mg}$ was $\mathrm{p}$ value of 0.968 .

\section{Postoperative time for the first analgesic request}

At the postoperative period, an independent $t$ test showed the time from the end of surgery to the first analgesic request was significantly different between TAP and non TAP groups $(p=0.000)$. The TAP block group showed as longer duration time for the first analgesic request than the controls with (mean $\pm \mathrm{SD})(286.00 \pm 166.31)$ vs (76.25 \pm 22.05$)$ minutes, correspondingly.

\section{Discussion}

The new type of peripheral regional anesthesia renowned as transversus abdominis plane (TAP) block have been stated in the management of multimodal analgesia for different types of abdominal Surgeries at the postoperative period. ${ }^{10,13,22}$ Largely studies demonstrate that the efficacy of TAP block as multimodal analgesia lowered postoperative severity of pain scores, reduced opioids consumption and complications as well as prolonged time for the first analgesic requests. ${ }^{23,24}$

The present study also showed that bilateral single injection of $20 \mathrm{ml}$ with $0.25 \%$ bupivacaine as multimodal analgesic regimen of TAP block resulted in lowered postoperative severity of pain, reduced Tramadol analgesic consumption near to $50 \%$, and a significant prolonged time for the first analgesic request in the first 24 postoperative hours as compared with the control group. The total Tramadol analgesics consumption reduction in the present study is comparable to Iraq which deals on TAP block after cesarean section delivery. This might be due to the same volume and percentage of bupivacaine used between our study and Iraq. The rationale for the reduced Tramadol consumption and long duration of analgesic effect of TAP blockade might be linked to the poorly vascularised TAP which allowed time-consuming clearance of bupivacaine local anaesthetic. ${ }^{3,22}$

However, the present results are incomparable to a study done on gynaecological malignancy surgery, which was no significant difference showed within the first 24 hours analgesics consumption and postoperative severity of pain on VAS measurement. This inconsistency to our study might be related with a high incidence of obesity in the study population resulting potentially technical failures. It is also the $27.6 \%$ of patients had incisions extended above the umbilicus. ${ }^{25}$ The TAP block through triangle of petit approach is documented as not to be successful higher than the umbilicus procedures even though it could be achieved by ultrasound guided sub costal TAP block technique. ${ }^{26}$ The abdominal wall which is the lateral side consists of three muscle layers, the external oblique, the internal oblique, and the transversus abdominis, and their respective fascial sheaths. ${ }^{2,3}$ The sensory afferent nerves pass through the transversus abdominis (neurofascial) plane (TAP) which is between the transversus abdominis and internal oblique muscle layer. Then the already deposited local anesthetic in this space is blocking the nerve roots originate from lumbar and thoracic plexuses region to lower severity of post surgical pain.,

There are limitations in our study. Since Randomized Control Trial (RCT) was not allowed in our Hospital, Patients' randomizations were not controlled. However, there were comparable between TAP block and control groups (Table 1). The participation of two BSC and one MSC anaesthetists both in TAP block and control groups might have an effect on the outcome of the study due to interpersonal dissimilarity of proficiency. We did not see the efficacy of TAP till 48 hours of postoperative period. It is difficult to say the intraoperative data collector is truly blinded, because of he might see the TAP block procedure at the end of the surgery.

\section{Conclusion and Recommendation}

In conclusion, a bilateral single injection of TAP block provides lower postoperative severity of pain, reduced total postoperative analgesics consumption and prolonged time for the first analgesic request as compared with non TAP block group after Cesarean Section upon under spinal anaesthesia when it is used as multimodal analgesia. We recommend TAP block should be included as part of multimodal analgesia in the postoperative period for women after Cesarean Section delivery. We also recommend further study to find out the effect of TAP block beyond 24 hours of postoperative period.

\section{Acknowledgments}

We would like to thank the study participants and Debre Tabore Hospital who made this study achievable.

\section{Conflicts of Interest}

The authors do not have any Conflict of interests.

\section{Funding}

None.

\section{References}

1. McMorrow RC, Ni Mhuircheartaigh RJ, Ahmed KA, et al. Comparison of transversus abdominis plane block vs spinal morphine for pain relief after Caesarean section. Br J Anaesth. 2011;106(5):706-712.

2. McDonnell JG, Curley G, Carney J, et al. The analgesic efficacy of transversus abdominis plane block after cesarean delivery: a randomized controlled trial. Anesth Analg. 2008;106(1):186-191.

3. Hamed Abed-Alnabi Flaifel, Muhalhi HA. postoperative analgesia of Transversus abdomonis plan block after cesarean section delivery under general anesthesia. Bas J Surg. 2013;35-43. 
4. Cali RL, Meade PG, Swanson MS, et al. Effect of Morphine and incision length on bowel function after colectomy. Dis Colon Rectum. 2000;43(2):163-168.

5. Tan TT, Teoh WH, Woo DC, et al. A randomised trial of the analgesic efficacy of ultrasoundguided transversus abdominis plane block after caesarean delivery under general anaesthesia. Eur $J$ Anaesthesiol. 2012;29: 88-94

6. Jørgensen H, Wetterslev J, Møiniche S, et al. Epidural local anaesthetics versus opioid-based analgesic regimens on postoperative gastrointestinal paralysis, PONV and pain after abdominal surgery. Cochrane Database Syst Rev. 2000;(4):CD001893.

7. Cameron CM, Scott DA, McDonald WM, et al. A review of neuraxial epidural morbidity: experience of more than 8,000 cases at a single teaching hospital. Anesthesiology. 2007;106(5): 997-1002.

8. Moen V, Irestedt L, Dahlgren N, et al. Major complications of central neuraxial block: the Third National Audit Project: some comments and questions. Br J Anaesth. 103(1):130-131.

9. McDonnell JG, O'Donnell B, Curley G, et al. The analgesic efficacy of transversus abdominis plane block after abdominal surgery: a prospective randomized controlled trial. Anesth Analg. 2007;104(1):193-197.

10. Conaghan P, Maxwell-Armstrong C, Bedforth N, et al. Efficacy of transversus abdominis plane blocks in laparoscopic colorectal resections. Surg Endosc. 2010;24(10):2480-2484.

11. McDonnell JG, O’Donnell BD, Farrell T, et al. Transversus abdominis plane block: a cadaveric and radiological evaluation. Reg Anesth Pain Med. 2007;32(5): 399-404.

12. O'Donnell BD, McDonnell JG, McShane AJ, et al. The transversus abdominis plane (TAP) block in open retropubic prostatectomy. Reg Anesth Pain Med. 2006;31(1): 91

13. Charlton S, Cyna AM, Middleton P, et al. (2010) Perioperative transversus abdominis plane (TAP) blocks for analgesia after abdominal surgery. Cochrane Database Syst Rev. 2010;(12):CD007705.

14. Wijewardana MGDG, Jayawardane MAMM. The Analgesic Efficacy of Transversus Abdominis Plane (TAP) Block after Total Abdominal Hysterectomy: A Randomized Controlled Trial. Sri Lanka Journal of Obstetrics and Gynaecology 6. 2014.

15. Madhumani NR, StevenCO, Aseem RH, et al. Determining Morphine Requirement Post Cesarean Section with and without TAP Block- A Retrospective Chart review. Int J Clin Anesthesiol. 2013;1(2):1012.
16. Young MJ, Gorlin AW, Modest VE, et al. Clinical implications of the transversus abdominis plane block in adults. Anesthesiol Res Pract. 2012: 731645

17. Fusco P, Scimia P, Paladini G, et al. Transversus abdominis plane block for analgesia after Cesarean delivery. A systematic review. Minerva Anestesiol. 2015;81(2):195-204.

18. Joselyn JKG, Bhalla T, Cambier G, et al. Providing effective perioperative analgesia with a unilateral Transversus Abdominis Plane (TAP) block in a patient with su- spected opioid allergie. Pediatric Anesthesia and Critical Care Journal. 2014;(2)1:40-43.

19. Belavy D, Cowlishaw PJ, Howes M, et al. (2009) Ultrasound-guided transversus abdominis plane block for analgesia after Caesarean delivery. Br J Anaesth. 2009;103(5):726-730.

20. Kumie FT, Gebremedhn EG, Tawuye HY, et al. Efficacy of fascia iliaca compartment nerve block as part of multimodal analgesia after surgery for femoral bone fracture. World J Emerg Med. 2015;6(2):142-146.

21. Degu G, Fasil T. Lecture notes for health science students biostatistics. University of Gondar 184. 2005.

22. Carney J, McDonnell JG, Ochana A, et al. The transversus abdominis plane block provides effective postoperative analgesia in patients undergoing total abdominal hysterectomy. Anesth Analg. 2008;107(6): 2056-2060.

23. Carney J, Finnerty O, Rauf J, et al. Ipsilateral transversus abdominis plane block provides effective analgesia after appendectomy in children: a randomized controlled trial. Anesth Analg. 2010;111(4): 998-1003.

24. Mishriky BM, George RB, Habib AS. Transversus abdominis planeblock for analgesia after Cesarean delivery a systematic review and meta analysis. Can J Anaesth. 2012;59(8):766-778.

25. Griffiths JD, Middle JV, Barron FA, et al. Transversus abdominis plane block does not provide additional benefit to multimodal analgesia in gynecological cancer surgery. Anesth Analg. 2010;111(3):797-801.

26. Tran TM, Ivanusic JJ, Hebbard P, et al. Determination of spread of injectate after ultrasound-guided transversus abdominis plane block: a cadaveric study. Br J Anaesth. 2009;102(1):123-127. 